\title{
ACRL meetings in San Antonio
}

A tentative schedule for the ALA

Midwinter Meeting in San

Antonio, January 19-25, 1996

- d. note: This list was current as we went to press. Be sure to check the program loook for final times and locations of meetings.

\section{ACRL Board of Directors}

Board/leader planning: Fridlay, Janulary 19 , 2:00-4:00 p.m. (closed)

First meeting: Sunday, January 21, 2:00-4:00 p.m.

Joint meeting with Budget \& Finance Committee: Monday, January 22, 10:00-11:00 a.m.

Second meeting: Tuesday, January 23, 2:005:30 p.m.

\section{ACRL General}

ACRL Presidential Candidates Forum and Reception: Saturday, January 20, 12:302:00 p.m.

Board/leader planning: Friday, January 19 , 2:00-4:00 p.m. (closed)

\section{ACRL Divisional Committees}

Academic/Research Librarian of the Year Award: Saturday, January 20. 8:30-11:00 a.m. (closed)

Academic Status: Sunday, January 21, 8:30-11:00 a.m.; Monday, January 22, 8:3011:00 a.m

Appointments and Nominations: Saturday. January 20, 8:30-1 1:00 a.m. ; Sunday. January 21, 2:00-4:00 p.m.; Tuesday, January 23, 8:00 a.m.-12:30 p.m. (closed)

Atkinson Award: Saturday, January 20, 8:009:00 a.m. (closed)

Budget and Finance: Saturclay, January 20, 8:30 a.m.-12:30 p.m.; Sunday, January $21,8: 30$ a.m.12:30 p.m.; Monday, January 22. 8:30-10:00 a.m.; Tuesday, January 23, 8:30-11:00 a.m.
Colleagues: Monday, January 22, 2:00-4:00 p.m.

Community Information Organizations: Monday, January 22, 8:30-11:00 à.m.

Conference Program Planning-New York, 1996: Saturday, January 20, 8:30-11:00 a.m.

Conference Program Planning-San Francisco, 1997: Saturday, January 20, 4:30-5:30 p.m.

Constitution and Bylaws: Saturday. January $20,11: 30$ a.m.-12:30 p.m.

Copyright: Sunclay, January 21, 8:30 a.m.12:30 p.m.

Council of Liaisons: Monday, January 22, 2:00-4:00 p.m.

Doctoral Dissertation Fellowship: Saturday. January 20, 9:30 a.m.-12:30 p.m.; Sunday, January 21, 2:00-4:00 p.m. (closed)

EASI Advisory: Sunday, January 21, 2:004:00 p.m.

Government Relations: Monday, January 22, 8:00-11:00 a.m.

Image Enhancement: Saturclay, January 20, 9:30-11:00 a.m.

Intellectual Freedom: Sunday, January 21 2:00-5:30 p.m.

International Relations: Sunday, January 21, 8:30-11:00 a.m.

K.G. Saur Award: Sunday, January 21, 8:009:00 a.m. (closed)

Lazerow Fellowship for Research in Acquisitions: Monday, January 22, 8:00-11:00 a.m. (closed)

Leadership Center Advisory: Saturdaly, January 20, 2:00-4:00 p.m.

Media Resources: Sunday, January 21, 9:3011:00 a.m.

Membership: Sunday, January 21, 4:30-5:30 p.m.

National Conference-Nashville: Saturday, January 20, 1:30-5:30 p.m.

Orientation: Saturday, January 20, 11:30 a.m.- 12:30 p.m.

President's Program-New York, 1996: 
Saturday, January 20, 8:30-11:00 a.m.; Sunday, January 21, 9:30-11:00 a.m.

President's Program Discussion Session: Saturday, January 20, 11:30 a.m.-12:30 p.m.

Professional Education: Sunday, January 21, 8:30-11:00 a.m.

Publications: Saturday, January 20, 8:00-9:00 a.m.; Monday, January 22, 8:00-11:30 a.m.

Racial and Ethnic Diversity: Saturday January 20, 9:30 a.m.-12:30 p.m.; Coalition: Saturday, January 20, 2:00-4:00 p.m.

Research: Saturday, January 20, 9:30-11:00 a.m.; Monday, January 22, 2:00-4:00 p.m.

Section Newsletter Editors: Monday, January $22,8: 00-11: 00$ a.m.

Standards and Accreditation: Saturday, January 20, 2:00-4:00 p.m.

Standards Study Task Force: Saturday, January 20, 9:30-11:00 a.m.

Statistics Committee: Sunday, January 21, 9:30-11:00 a.m.; Monday, January 22, 9:30-11:00 a.m.

\section{ACRL Chapters Council}

ACRL Presidential Candidates Forum/Reception: Saturday, January 20, 12:30-2:00 p.m.

Business and Orientation: Sunday, January $21,8: 30$ a.m. $-12: 30$ p.m.

\section{ACRL Editorial Boards}

Choice: Sunday, January 21, 9:30 a.m.-12:30 p.m. College \& Research Libraries: Saturday, January 20, 4:30-5:30 p.m.

College \& Research Libraries News: Sunday, January 21, 9:30 a.m.-12:30 p.m.

\section{Meet the candidates for ACRL president}

Hear Bernard Fradkin and W. Lee Hisle, the official candidates for ACRL vice-president/president-elect, speak at the ACRL Presidential Candidates Forum on Saturday, January 20, 1996, 12:30-2:00 P.m., during the ALA Midwinter Meeting in San Antonio. Fradkin is dean of the Learning Resources Center at the College of DuPage in Glen Ellyn, Illinois. Hisle is dean of learning resources services at Austin Community College in Texas. The Forum, organized by the ACRL Chapters Council, is followed by a reception sponsored by EBSCO Subscription Services.
New Publications Advisory Board: Sunday, January $21,11: 30$ a.m. $-12: 30$ p.m.

New Publications Advisory Board Graphics Subcommittee: Saturday, January 20, 8:009:00 a.m

Publications in Librariansbip: Sunday, January 21, 8:00-9:00 a.m.

Rare Books \& Manuscripts Librarianship: Saturday, January 20, 8:00-10:00 p.m.

\section{ACRL Sections}

Activity Sections Council: Friday, January 19, 4:30-5:30 p.m.

\section{Afro-American Studies Librarian Section}

Executive: Saturday, January 20, 2:00-4:00 p.m.

Business/Membership: Monday, January 22, 9:30-11:00 a.m.

Indexing Project: Saturday, January 20, 8:3011:00 a.m.

Standing Committees: Saturday, January 20, 8:30-11:00 a.m.

\section{Asian, African and Middle Eastern Section}

Executive: Sunday, January 21, 2:00-4:00 p.m.

\section{Anthropology and Sociology Section}

Executive: Saturday, January 20, 2:00-4:00 p.m.; Monday, January 22, 2:00-4:00 p.m.

Anthropology Librarians Discussion Group: Sunday, January 21, 4:30-5:30 p.m.

Bibliography: Saturday, January 20, 9:30 11:00 a.m.; Monday, January 22, 8:30-11:00 a.m.

Conference Program Planning-New York, 1996: Saturday, January 20, 4:30-5:30 p.m.

Conference Program Planning-San Francisco, 1997: Sunday, January 21, 8:00-9:00 a.m.;

Liaison: Sunday, January 21, 9:30-11:00 a.m.

Publications: Sunday, January 21, 2:00-4:00 p.m.

Review and Planning: Sunday, January 21, 11:30 a.m. $-12: 30$ p.m.

Sociology Librarians Discussion Group: Saturday, January 20, 11:30 a.m.-12:30 p.m.

\section{Arts Section}

Executive and Membership: Sunday, January $21,9: 30$ a.m.- $12: 30$ p.m.

All Committees: Saturday, January 20, 9:30 a.m.-12:30 p.m.

Dance Librarians Discussion Group: Sunday, January 21, 2:00-4:00 p.m. 
Dance Video/Interlibrary Loan: Saturday, January 20, 8:00-9:00 a.m.

Film and Broadcast Studies Discussion Group: Monday, January 22, 2:00-4:00 p.m.

Technology in the Arts: Monday, January 22, 8:30-11:00 a.m.

\section{Community and Junior College Libraries Section}

Executive: Saturday, January 20, 11:30 a.m.-12:30 p.m.; Monday, January 22, 9:3011:00 a.m.

All Committees: Saturday, January 20, 2:004:00 p.m.

Awards: Sunday, January 21, 8:30-11:00 a.m. (closed)

CJCLS/NCRL Joint Discussion Group: Sunday, January 21, 2:00-4:00 p.m.

Conference Program Planning-San Francisco, 1997: Sunday, January 21, 9:30-11:00 a.m.

Local Arrangements Planning: Sunday, January $21,9: 30-11: 00$ a.m.

\section{College Libraries Section}

Executive: Saturday, January 20, 9:30 a.m.12:30 p.m.; Tuesday, January $23,9: 30$ a.m.-12:30 p.m.

CLIP Notes: Saturday, January 20, 9:30-11:00 a.m.

CLS Standards: Friday, January 19, 2:00-4:00 p.m.; Monday, January 22, 9:30-11:00 a.m.

College Libraries Discussion Forum: Monday, January 22, 9:30-11:00 a.m.

College Library Directors' Discussion Group: Sunday, January 21, 2:00-4:00 p.m.

College Library Leadership: Monday, January $22,8: 00-9: 00$ a.m.

Continuing Education: Saturday, January 20, 2:00-4:00 p.m.

Medium-Sized Academic Libraries Discussion Group: Saturday, January 20, 2:00-4:00 p.m.

Newsletter: Saturday, January 20, 2:00-4:00 p.m.

Research for College Librarianship: Sunday, January 21, 9:30 a.m.-12:30 p.m. Topic: "Topics, Tapes and Techniques for College Librarians Submitting Papers and Panel Presentations in Nashville and Beyond."

Women's Colleges Discussion Group: Saturday, January 20, 2:00-4:00 p.m.

\section{English and American Literature Section}

Executive: Saturday, Jantary 20, 2:00-4:00 p.m.
General Membership Meeting: Sunday, January $21,9: 30$ a.m. $-12: 30$ p.m.

All Committees: Monday, January 22, 8:0011:00 a.m.

\section{Education and Behavioral Sciences Section}

Executive: Friday, January 19, 8:00-10:00 p.m.

Advisory Board: Sunday, January 21, 2:005:00 p.m.

Bibliographic Instruction for Educators: Saturday, January 20, 2:00-4:00 p.m.

Conference Program Planning-New York, 1996: Saturday, January 20, 2:00-4:00 p.m.

Current Topics Discussion Group: Sunday, January 21, 9:00-11:00 a.m.

Curriculum Materials/Centers Management: Saturday, January 20, 2:00-4:00 p.m.

Distinguished EBSS Librarian Award: Saturday, January 20, 8:00-9:00 a.m. (closed)

Government Policy: Saturday, January 20, 2:00-4:00 p.m.

Membership and Orientation: Saturday, January 20, 9:00-11:00 a.m.

Nominating 1996: Saturday, January 20, 8:009:00 a.m. (closed)

Nominating 1997: Saturday, January 20, 8:009:00 a.m. (closed)

Problem of Access \& Control of Educational Materials/Curriculum Materials Centers: Saturday, January 20, 9:00-11:00 a.m.

Psychology and Psychiatry: Saturday, January 20, 9:00-11:00 a.m.

Publications: Saturday, January 20, 8:00-9:00 a.m.

Reference Services: Saturday, January 20, 9:00-11:00 a.m.

Social Work/Social Welfare: Friday, January 19, 2:00-4:00 p.m.

\section{Extended Campus Library Services Section}

Executive: Saturday, January 20, 8:0010:00 p.m.; Sunday, January $21,11: 30$ a.m.12:30 p.m.

All Committees: Sunday, January 21, 8:3011:00 a.m.

Discussion Group/General Membership: Monday, January 22, 8:30-11:00 a.m.

Planning: Monday, January 22, 2:00-4:00 p.m.

\section{Instruction Section}

Executive: Friday, January 19, 9:30 a.m.-12:30 p.m.; Monday, January 22, 2:00-4:00 p.m. 
Advisory Council: Saturday, January 20, 2:005:30 p.m.; Monday, January 22, 8:30-11:00 a.m.

Awards: Saturday, January 20, 8:30-11:00 a.m.; Sunday, January 21 , 9:30 a.m.-12:30 p.m. (closed)

Communications: Saturday, January 20, 8:009:00 a.m.; Sunday, January 21, 8:00-9:00 a.m. and 2:00-4:00 p.m.

Conference Program Planning - San Francisco, 1997: Saturday, January 20, 9:30-11:00 a.m.; Sunday, January 21, 9:30-11:00 a.m.

Conference Program Planning-New York, 1996: Sunday, January 21, 9:30-11:00 a.m.

Continuing Education: Saturday, January 20, 9:30-11:00 a.m.; Sunday, January 21, 9:30-11:00 a.m.

Discussion Forum: Sunday, January 21, 4:305:30 p.m.

Education for Bibliographic Instruction: Sunday, January 21, 9:30-11:00 a.m.

Education for Library Instruction: Saturday, January 20, 9:30-11:00 a.m.

Emerging Technologies in Instruction: Saturday, January 20, 9:30-11:00 a.m.; Sunday, January 21, 9:30-11:00 a.m.

Guidelines for Bibliographic Instruction: Saturday, January 20,9:30-11:00 a.m. and 11:30a.m.-12:30 p.m.; Sunday, January 21, 9:3011:00 a.m.

Instruction for Diverse Populations: Saturday, January 20, 9:30-11:00 a.m.; Sunday, January $21,9: 30-11: 00$ a.m.

Knowledge Based Task Force: Saturday, January 20, 9:30-11:00 a.m.; Sunday, January 21, 9:30-11:00 a.m.

Management of Bibliographic Instruction Services: Saturday, January 20, 9:30-11:00 a.m.; Sunday, January 21, 9:30-11:00 a.m.

Membership: Saturday, January 20, 9:3011:00 a.m.; Sunday, January 21, 9:30-11:00 a.m.

Name Change Implementation Task Force: Saturday, January 20, 9:30-11:00 a.m.; Sunday, January 21, 9:30-11:00 a.m.

Nominating 1996: Saturday, January 20, 8:30-

11:00 a.m. (closed)

Nominating 1997: Saturday, January 20, 8:3011:00 a.m. (closed)

l'lanning: Saturday, January 20, 9:30-11:00 a.m. Policy: Saturday, January 20, 9:30-11:00 a.m.; Sunday, January 21, 9:30-11:00 a.m.

Preconference Planning 1997: Saturday, January 20, 9:30-11:00 a.m.

Teaching Methods: Saturday, January 20, 9:30-11:00 a.m.; Sunday, January 21, 11:30 a.m.$12: 30$ p.m.

\section{Law and Political Sciences Section}

Executive: Saturday, January 20, 9:30 a.m.10:30 a.m.

Conference Program Planning-San Francisco, 1997: Saturday, January 20, 10:30 a.m. 12:30 p.m.

General Membership: Monday, January 22, 9:30-11:30 a.m.

Library Instruction: Sunday, January 21, 2:004:00 p.m.

Marta Lange Award: Sunday, January 21, 11:30 a.m.-12:30 p.m.

Membership: Saturday, January 20, 10:30 a.m.12:30 p.m.

Publications: Saturday, January 20, 10:30 a.m.12:30 p.m.

Review and Planning: Saturday, January 20, 10:30 a.m. $-12: 30$ p.m.

Vendor/Publisher Relations: Saturday, January $20,10: 30$ a.m.-12:30 p.m.

\section{Rare Books and Manuscripts Section}

Executive: Sunday, January 21, 2:00-4:00 p.m.; Monday, January 22, 8:30-11:00 a.m.

Bibliographic Standards: Saturday, January 20, 8:30-11:00 a.m.; Sunday, January 21, 8:3011:00 a.m.

Budget and Development: Saturday, January 20, 2:00-4:00 p.m.

Conference Development: Sunday, January 21, 8:30-11:00 a.m.

Conference Program Planning - San Francisco, 1997: Sunday, January 21, 11:30 a.m.-12:30 p.m.

Curators and Conservators Discussion Group: Sunclay, January 21, 8:30-11:00 a.m.

Education and Professional Development: Saturday, January 20, 2:00-4:00 p.m.

Electronic Resources ad hoc: Sunday, January 21, 8:30-11:00 a.m.

Exhibition Catalog Awards: Saturday, January 20, 9:30 a.m-12:30 p.m.; Sunday, January 21, 9:30 a.m-12:30 p.m (closed)

Information Exchange: Sunday, January 21, 4:30-5:30 p.m.

Manuscripts and Other Formats Discussion Group: Saturday, January 20, 8:30-11:00 a.m.

MARC for Special Collections (MASC) Discussion Group: Sunday, January 21, 11:30 a.m.12:30 p.m.

Membership ad hoc: Saturday, January 20, 2:00-4:00 p.m.

Nominating: Saturday, January 20, 11:30 a.m.12:30 p.m. (closed)

Preconference Program Planning-New 
York, 1996: Sunday, January 21, 11:30 a.m.12:30 p.m.

Preconference Program Planning-San Francisco, 1997: Saturday, January 20, 4:30-5:30 p.m.

Publications: Saturday, January 20, 9:30-11:00 a.m.

Security: Saturday, January 20, 9:30-11:00 a.m

Seminars: Saturday, January 20, 8:00-10:00 p.m.

\section{Slavic and East European Section}

Executive: Sunday, January 21, 4:30-5:30 p.m.

Automated Bibliographic Control: Sunday, January 21, 2:00-4:00 p.m.

Continuing Education on Slavic and East European Librarianship in North America: Sunday, January 21, 9:30-11:00 a.m.

Newsletter Editorial Board: Sunday, January 21, 11:30 a.m.-12:30 p.m.

Nominating: Saturday, January 20, 4:30-5:30 p.m. (closed)

Preservation of Slavic Materials and Special Collections: Saturday, January 20, 2:00-4:00 p.m.

\section{Science and Technology Section}

Comparison of Science and Technology Libraries: Sunday, January 21, 9:30 a.m.-12:30 p.m.

Conference Program Planning-New York, 1996: Saturday, January 20, 4:30-5:30 p.m.

Conference Program Planning-San Francisco, 1997: Sunday, January 21, 9:30 a.m.-12:30 p.m.

Continuing Education: Sunday, January 21, 9:30 a.m.-12:30 p.m.

Council: Friday, January 19, 8:00-10:00 p.m.; Monday, January 22, 2:00-4:00 p.m.

Discussion Group Leaders: Saturday, January 20, 9:30 a.m.-12:30 p.m.

Forum for Science and Technology Library Research: Sunday, January 21, 8:30-11:00 a.m.

General Discussion Group: Sunday, January $21,2: 00-4: 00$ p.m.

Heads of Science and Technology Libraries Discussion Group: Sunday, January 21, 8:00-10:00 p.m.

Legislation: Saturday, January 20, 8:00-10:00 p.m.; Monday, January 22, 8:30-11:00 a.m.

Membership and Recruitment: Saturday, January 20, 9:30 a.m.-12:30 p.m.

Oberly Award: Saturday, January 20, 9:30 a.m.-12:30 p.m. (closed)

Organization and Planning: Sunday, January $21,9: 30$ a.m.-12:30 p.m.

Publications: Saturday, January 20, 9:30 a.m.12:30 p.m.
Publisher/Vendor Relations: Saturday, January 20, 9:30 a.m.-12:30 p.m.

Science and Technology Databases Discussion Group: Saturday, January 20, 2:00-4:00 p.m.

Subject and Bibliographic Access: Saturday, January 20, 9:30 a.m.-12:30 p.m.

\section{University Libraries Section}

Executive: Saturday, January 20, 9:30-11:00 a.m.; Monday, January 22, 9:30-11:00 a.m.

Communications: Saturday, January 20, 11:30 a.m. $-12: 30$ p.m.

Current Topics: Saturday, January 20, 2:004:00 p.m.

Libraries in Higher Education Discussion Group: Sunday, January 21, 9:30-11:00 a.m.

Nominating: Saturday, January 20, 8:00-9:00 a.m. (closed)

Organization and Bylaws: Sunday, January 21, 2:00-4:00 p.m.

Policy and Planning: Sunday, January 21, 9:3011:00 a.m.

Public Service Directors of Large Research Libraries Discussion Group: Sunday, January 21, 2:00-4:00 p.m.

\section{Western European Specialists Section}

Executive: Tuesday, January 23, 11:30 a.m. 1:00 p.m.

Classical, Medieval, and Renaissance Discussion Group: Saturday, January 20, 2:00-4:00 p.m.

College and Medium-Sized Libraries Discussion Group: Monday, January 22, 8:00-9:00 a.m.

Conference Program Planning-New York, 1996: Saturday, January 20, 2:00-4:00 p.m.

General Membership Meeting and Discussion Group: Monday, January 22, 9:30-11:00 a.m.

Germanists Discussion Group: Sunday, January $21,9: 30-11: 00$ a.m.

\section{Address the ACRL Board}

Share your concerns directly with ACRL's Board of Directors. The first 21 minutes of the Sunday, January 21, 1996, meeting of the ACRL Board in San Antonio will be given to an open-mike period. ACRL members may-on a first-come, first-served basisaddress the Board (with a limit of three minutes per speaker) on any topic. The Board hopes this communication method will facilitate hearing the concerns and interests of ACRL members. 
German Social Sciences Working Group: Sunday, January 21, 8:00-9:00 a.m.

Internet Publications Committee: Tuesday, January 22, 8:00-9:00 a.m.

Nijhoff Award Committee: Sunday, January 21, 4:30-5:30 p.m. (closed)

Preconference Planning Committee-San Francisco, 1997: Saturday, January 20, 8:00-10:00 p.m.

Publications: Tuesday, January 23, 9:30-11:00 a.m.

Research and Planning: Sunday, January 21, 2:00-4:00 p.m.

Romance Languages Discussion Group:
Monday, January 22, 2:00-4:00 p.m.

Scandinavian Discussion Group: Sunday, January 21, 4:30-5:30 p.m.

\section{Women's Słudies Section}

Executive: Saturday, January 20, 2:00-4:00 p.m.; Monday, January 22, 2:00-4:00 p.m.

All Committees: Sunday, January 21, 9:3011:00 a.m.

Discussion Meeting: Sunday, January 21, 11:30 a.m.- 12:30 p.m.

General Membership: Monday, January 22, 9:30-11:00 a.m.

\section{ACRL Discussion Groups}

$E d$. Note: Section discussion groups are listed with their sections.

Alliances for New Directions in Teaching/Learning: Monday, January 22, 8:00-9:00 a.m. Topic: "National Programs to Promote the Use of Information Technology in Higher Education Teaching and Learning."

Australian Studies: Sunday, January 21, 11:30 a.m.-12:30 p.m. Topic: "International Business Resources."

Canadian Studies: Saturday, January 20 . 11:30 a.m.-12:30 p.m. Topic: "Canada in the North American Context."

Electronic Library Development in Academic Libraries: Sunday, January 21, 9:30-11:00 a.m. Topic: "Libraries and Computing Unite for Service in the Next Century."

Electronic Reserves: Sunday, January 21. 8:30-11:00 a.m. Topic: "An Open Discussion on Ulsing Electronic Reseries Systems."

Electronic Text Centers: Saturday, January 20, 2:00-4:00 p.m. Topic: "User Education and Outreach for the ETC: What Works."

Exhibits and Displays in College Libraries: Saturday, January 20, 9:30-11:00 a.m. Topic: "Tour and Discussion at the Institute for Texan Culture."

Fee-based Information Service Centers in Academic Libraries (FISCAL): Saturday, January 20, 2:00-4:00 p.m. Topic: "Marketing Fee-Based Information Services Using the Internet"; Sunday, January 21, 9:30-11:00 a.m.

Fund-Raising and Development: Sunday, January 21, 9:30-11:00 a.m. Topic: "Bread and Butter Fund-Raising. "

Heads of Public Services: Sunday, Janu- ary 21, 4:30-6:00 p.m. Topic: "Teaching Information Literacy to Undergraduate Students."

Home Economics/Human Ecology Librarians: Sunday, January 21, 11:30 a.m. 12:30 p.m. Topic: "Creating a Home Page on the $W W W$."

Journal Costs in Academic Libraries: Saturday, January 20, 2:00-4:00 p.m. Topic: "Partnerships: Reality or Myth?"

Library Development Officers: Sunday, January 21, 9:30-11:00 a.m.

MLA International Bibliography in Academic Libraries: Saturday, January 20, 8:0011:00 a.m. Topic: "Using the MLA Bibliography for Collection Development in Academic Libraries."

Personnel Administrators and Staff Development Officers of Large Research Libraries: Saturday, January 20, 8:30 a.m.-12:30 p.m. Topic: "Facilitating Organizational Change: The Human Resources Role." ; Sunday, January 21, 8:30 a.m.-12:30 p.m.

Philosophical, Religious, and Theological Studies: Monday, January 22, 9:30-11:00 a.m

Popular Culture and Libraries: Sunday, January $21,4: 30-5: 30$ p.m.

Public Relations in Academic Libraries: Sunday, January 21, 2:00-4:00 p.m. Topic: "Neusletters That Work: Making the Most of Your Time and Money."

Undergraduate Librarians: Monday, January 22, 8:30-11:00 a.m. Topic: Senvice Innovation Discussion. 


\title{
Ginally?
}

\section{Upgraded CIP Records At the time of publication!}

\author{
Available for Approval \\ and New Titles Books \\ from \\ BLACKWELL \\ NORTH AMERICA
}

Where Innovation is a Tradition

TECHSERV@BNAMF.BLACKWELL.COM 